\title{
A Machine Learning Approach to Automatic Music Genre Classification
}

\author{
Carlos N. Silla Jr. ${ }^{1}$, Alessandro L. Koerich ${ }^{2}$ and Celso A. A. Kaestner ${ }^{3}$ \\ ${ }^{1}$ University of Kent - Computing Laboratory \\ Canterbury, CT2 7NF \\ Kent, United Kingdom \\ cns2@kent.ac.uk \\ ${ }^{2}$ Pontifical Catholic University of Paraná \\ R. Imaculada Conceição 1155, 80215-901 \\ Curitiba - Paraná - Brazil \\ alekoe@ppgia.pucpr.br \\ ${ }^{3}$ Federal University of Technology of Paraná \\ Av. Sete de Setembro 3165, 80230-901 \\ Curitiba - Paraná - Brazil \\ celsokaestner@utfpr.edu.br
}

Received 06 January 2008; accepted 23 July 2008

\begin{abstract}
This paper presents a non-conventional approach for the automatic music genre classification problem. The proposed approach uses multiple feature vectors and a pattern recognition ensemble approach, according to space and time decomposition schemes. Despite being music genre classification a multi-class problem, we accomplish the task using a set of binary classifiers, whose results are merged in order to produce the final music genre label (space decomposition). Music segments are also decomposed according to time segments obtained from the beginning, middle and end parts of the original music signal (time-decomposition). The final classification is obtained from the set of individual results, according to a combination procedure. Classical machine learning algorithms such as Nä̈ve-Bayes, Decision Trees, $k$ Nearest-Neighbors, Support Vector Machines and MultiLayer Perceptron Neural Nets are employed. Experiments were carried out on a novel dataset called Latin Music Database, which contains 3,160 music pieces categorized in 10 musical genres. Experimental results show that the proposed ensemble approach produces better results
\end{abstract}

than the ones obtained from global and individual segment classifiers in most cases. Some experiments related to feature selection were also conducted, using the genetic algorithm paradigm. They show that the most important features for the classification task vary according to their origin in the music signal.

Keywords: music genre classification, machine learning, pattern classification, feature selection.

\section{INTRODUCTION}

Music is nowadays a significant part of the Internet content: the net is probably the most important source of music pieces, with several sites dedicated to spreading, distributing and commercializing music. In this context, automatic procedures capable of dealing with large amounts of music in digital formats are imperative, and Music Information Retrieval (MIR) has become an important research area.

One of the tasks focused by MIR is the Automatic Music Genre Classification (AMGC) problem. In essence music genres are categorical labels created by human ex- 
perts in order to identify the style of the music. The music genre is a descriptor that is largely used to organize collections of digital music. It is not only a crucial metadata in large music databases and electronic music distribution (EMD) [1], but also the most frequent item used in search queries, as pointed by several authors [8], [20], [30], [35], [36].

Up to now the standard procedure for organizing music content is the manual use of meta information tags, such as the ID3 tags associated to music coded in MPEG-1 Audio Layer 3 (MP3) compression format [15]. This metadata includes song title, album, year, track number and music genre, as well as other specific details of the file content. The music genre information, however, is usually incomplete and inaccurate, since it depends on human subjectiveness. As there is no industrial standard, more confusion arises: for example, the same music piece is indicated as belonging to different music genres according to different EMD sites, due to different interpretation and/or different codification schemes [19].

We emphasize that AMGC also poses an interesting research problem from a pattern recognition perspective. Music can be considered as a high-dimensional digital time-variant signal, and music databases can be very large [2]; thus, it offers a good opportunity for testing nonconventional pattern recognition approaches.

As each digital music segment can be associated to a vector representative, by applying some extracting procedure to calculate appropriate feature values, to put this problem as a classical classification task in the pattern recognition framework is straightforward [28]. Typically a music database (as in a EMD) contains thousands of pieces from dozens of manually-defined music genres [1], [23], [32] characterizing a complex multi-class problem.

In this paper we present a non-conventional machine learning approach to the AMGC problem. We use the ensemble approach [7], [17] based on both space and time decomposition schemes. Firstly, the AMGC problem is naturally multi-class; as pointed in the literature [7], [12], inter class similarity / extra class distinction are inversely proportional to the number of classes involved. This situation can be explained by considering that is difficult to most classifiers to construct an adequate separation surface among the classes [24]. One solution is to decompose the original multi-class problem space as a series of binary classification problems, where most of the known classifiers work better, and to merge the obtained results in order to produce the final result. Secondly, as music is a time-varying signal, several segments can be employed to extract features, in order to produce a set of feature vectors that characterizes a decomposition of the original signal according to the time dimension. We employ an ensemble approach that encompasses both decompositions: classifiers are applied to space-time partial views of the music, and the obtained classification results are merged to produce the final class label.

We also conduct some experiments related to feature selection, employing a genetic algorithm framework, in order to show the relation between the relative importance of each feature and its origin in the music signal.

This paper is organized as follows: section 2 presents a formal view of the problem and summarize the results of several research works in automatic music genre classification; section 3 presents our proposal, based on spacetime decomposition strategies, describing also the employed features, classification and results combination algorithms; section 4 presents the employed dataset and the experimental results obtained with the space-time decomposition; section 5 describes the employed feature selection procedure, and the obtained experimental results; finally, section 6 presents the conclusions of our work.

\section{Problem definition AND RElated WORKS}

Nowadays the music signal representation is no longer analogous to the original sound wave. The analogical signal is sampled, several times per second, and transformed by an analogous-to-digital converter into a sequence of numeric values in a convenient scale. This sequence represent the digital audio signal of the music, and can be employed to reproduce the music [15].

Hence the digital audio signal can be represented by a sequence $S=<s_{1}, s_{2} \ldots s_{N}>$ where $s_{i}$ stands for the signal sampled in the instant $i$ and $N$ is the total number of samples of the music. This sequence contains a lot of acoustic information, and features related to timbral texture, rhythm and pitch content can be extracted from it. Initially the acoustic features are extracted from short frames of the audio signal; then they are aggregated into more abstract segment-level features [2]. So a feature vector $\bar{X}=<x_{1}, x_{2} \ldots x_{D}>$ can be generated, where each feature $x_{j}$ is extracted from $S$ (or some part of it) by an appropriate extraction procedure.

Now we can formally define the AMGC problem as a pattern classification problem, using segment-level features as input. From a finite set of music genres $\mathcal{G}$ we must select one class $\hat{g}$ which best represents the genre of the music associated to the signal $S$.

From a statistical perspective the goal is to find the most likely $\hat{g} \in \mathcal{G}$, given the feature vector $\bar{X}$, that is

$$
\hat{g}=\arg \max _{g \in G} P(g \mid \bar{X})
$$

where $P(g \mid \bar{X})$ is the a posteriori probability that the music belong to the genre $g$ given the features expressed by $\bar{X}$. Using the Bayes' rule the equation can be rewritten as

$$
\hat{g}=\arg \max _{g \in G} \frac{P(\bar{X} \mid g) \cdot P(g)}{P(\bar{X})}
$$


where $P(\bar{X} \mid g)$ is the probability in which the feature vector $\bar{X}$ occurs in class $g, P(g)$ is the a priori probability of the music genre $g$ (which can be estimated from frequencies in the database) and $P(\bar{X})$ is the probability of occurrence of the feature vector $\bar{X}$. The last probability is in general unknown, but if the classifier computes the likelihoods of the entire set of genres, then $\sum_{g \in \mathcal{G}} P(g \mid \bar{X})=1$ and we can obtain the desired probabilities for each $g \in \mathcal{G}$ by

$$
P(g \mid \bar{X})=\frac{P(\bar{X} \mid g) \cdot P(g)}{\sum_{g \in \mathcal{G}} P(\bar{X} \mid g) \cdot P(g)}
$$

The AMGC problem was initially defined in the work of Tzanetakis and Cook [35]. In this work a comprehensive set of features was proposed to represent a music piece. These features are obtained from a signal processing perspective, and include timbral texture features, beat-related features and pitch-related features. As classification procedures they employ Gaussian classifiers, Gaussian mixture models and the $k$ Nearest-Neighbors $(k$-NN) classifier. The experiments occur in a database called GTZAN, that include 1,000 samples from ten music genres, with features extracted from the first 30seconds of each music. Obtained results indicate an accuracy of about $60 \%$ using a ten-fold cross validation procedure. The employed feature set has become of public use, as part of the MARSYAS framework (Music Analysis, Retrieval and SYnthesis for Audio Signals, available at http://marsyas.sourgeforge.net/), a free software platform for developing and evaluating computer audio applications [35].

Kosina [19] developed MUGRAT (MUsic Genre Recognition by Analysis of Texture, available at http://kyrah.net/mugrat), a prototypical system for music genre recognition based on a subset of the features given by the MARSYAS framework. In this case the features were extracted from 3 -second segments randomly selected from the entire music signal. Experiments were carried out in a database composed by 186 music samples belonging to 3 music genres. Employing a 3-NN classifier Kosina obtains an average accuracy of $88.35 \%$ using a ten-fold cross-validation procedure. In this work the author also confirms that manually-made music genre classification is inconsistent: the very same music pieces obtained from different EMD sources were differently labeled in their ID3 genre tag.

Li, Ogihara and Li [21] present a comparative study between the features included in the MARSYAS framework and a set of features based on Daubechies Wavelet Coefficient Histograms (DWCH), using also other classification methods such as Support Vector Machines (SVM) and Linear Discriminant Analysis (LDA). For comparison purposes they employ two datasets: (a) the original dataset of Tzanetakis and Cook (GTZAN), with features extracted from the beginning of the music signal, and (b) a dataset composed by 755 music pieces of 5 music genres, with features extracted from the interval that goes from second 31 to second 61. Conducted experiments show that the SVM classifier outperforms all other methods: in case (a) it improves accuracy to $72 \%$ using the original feature set and to $78 \%$ using the DWCH feature set; in case (b) the results were $71 \%$ for the MARSYAS feature set and $74 \%$ to the DWCH feature set. The authors also evaluate some space decomposition strategies: the original multi-class problem (5 classes) was decomposed in a series of binary classification problems, according to a One-Against-All (OAA) and Round-Robin (RR) strategies (see Section 3.1). The best results were achieved with SVM and OAA space decomposition using DWCH feature set. Accuracy was improved by 2 to $7 \%$ according to employed feature set - DWCH and MARSYAS respectively - in the dataset (a), and by 2 to $4 \%$ in dataset (b).

Grimaldi, Cunningham and Kokaram [13], [14] employ a space decomposition strategy to the AMGC problem, using specialized classifiers for each space vision and an ensemble approach to obtain the final classification decision. The authors decompose the original problem according to OAA, RR - called pairwise comparison [12] - and random selection of subspaces [16] methods. They also employ different feature selection procedures, such as ranking according to information gain (IG) and gain ratio (GR), and Principal Component Analysis (PCA). Experiments were conducted in a database of 200 music pieces of 5 music genres, using the $k$-NN classifier and a 5-fold cross validation procedure. The feature set was obtained from the entire music piece, using discrete wavelet transform (DPWT). For $k$-NN classifier the PCA analysis proves to be the most effective feature selection technique, achieving an accuracy of $79 \%$. The RR ensemble approach scores $81 \%$ for both IG and GR, showing to be an effective ensemble technique. When applying a forward sequential feature selection based on the GR ranking, the ensemble scores $84 \%$.

The work of Meng, Ahrendt and Larsen [27] deals with the relative importance of the features. They employ features based on three time scales: (a) short-term features, computed over 30 milliseconds windows, and related to timbral texture; (b) middle-term features, obtained from 740 milliseconds windows and related to modulation and/or instrumentation; and (c) long-term features, computed over 9.62 seconds windows and related to beat pattern and rhythm. They use two classifiers: a single layer neural net and a Gaussian classifier based on the covariance matrix. They performed experiments on two datasets, the first one with 100 music pieces from 5 music genres and the second with 354 music pieces from $6 \mathrm{mu}-$ sic genres. The evaluation of the experiments shows that a combination of middle and long-term features produce 
better results for classification.

Yaslan and Catalpete [38] employ a large set of classifiers to study the problem of feature selection in the AMGC problem. They use the linear and quadratic discriminant classifiers, the Naïve-Bayes classifier, and variations of the $k$-NN classifier. They employ the GTZAN database and the MARSYAS framework [35] for feature extraction. The features were analyzed according to groups, for each one of the classifiers. They employ the Forward Feature Selection (FFS) and Backward Feature Selection (BFS) methods, in order to find the best feature set for the problem. These methods are based on guided search in the feature space, starting from the empty set and from the entire set of features, respectively. They report positive results in the classification with the use of the feature selection procedure.

Up to now most researchers attack the AMGC problem by ensemble techniques using only space decomposition. We emphasize that these techniques employ different views of the feature space and classifiers dedicated to these subspaces to produce partial classifications, and a combination procedure to produce the final class label. Furthermore the features used in these works are selected from one specific part of the music signal or from the entire music signal.

One exception is the work of Bergstra et al. [2]. They use the ensemble learner AdaBoost [11] which performs the classification iteratively by combining the weighted votes of several weak learners. Their model uses simple decision stumps, each of which operates on a single feature dimension. The procedure shows to be effective in three music genre databases (Magnatune, USPOP and GTZAN), winning the music genre identification task in the MIREX 2005 (Music Information Retrieval EXchange) [9]. Their best accuracy results vary from 75 to $86 \%$ in these databases.

The first work that employs time decomposition using regular classifiers applied to complete feature vectors was proposed by Costa, Valle Jr. and Koerich [4]. This work presents experiments based on ensemble of classifiers approach that uses three time segments of the music audio signal, and where the final decision is given by the majority vote rule. They employ a MLP neural net and the $k$-NN classifiers. Experiments were conducted on a database of 414 music pieces of 2 genres. However, final results regarding the quality of the method for the classification task were inconclusive. Koerich and Poitevin [18] employ the same database and an ensemble approach with a different set of combination rules. Given the set of individual classifications and their corresponding score a number associated to each class also obtained from the classification procedure - they use the maximum, the sum and pondered sum, product and pondered product of the scores to assign the final class. Their experiments show better results than the individual classifications when using two segments and the pondered sum and the pondered product as result combination rules.

\section{THE SPACE-TIME DECOMPOSITION APPROACH}

In this paper we evaluate the effect of using the ensemble approach in the AMGC problem, where individual classifiers are applied to a special decomposition of the music signal that encompasses both space and time dimensions. We use feature space decomposition following the OAA and RR approaches, and also features extracted from different time segments [31], [33], [34]. Therefore several feature vectors and component classifiers are used in each music part, and a combination procedure is employed to produce the final class label for the music.

\subsection{SPACE DECOMPOSITION}

Music genre classification is naturally a multi-class problem. However, we employ a combination of the results given by binary classifiers, whose results are merged afterwards in order to produce the final music genre label.

This procedure characterizes a space decomposition of the feature space, since features are used according to different views of the problem space. The approach is justified because for two class problems the classifiers tend to be simple and effective. This point is related to the type of the separation surface constructed by the classifier, which are limited in several cases [24].

Two main techniques are employed to produce the desired decomposition: (a) in the one-against-all (OAA) approach, a classifier is constructed for each class, and all the examples in the remaining classes are considered as negative examples of that class; and (b) in the round-robin (RR) approach, a classifier is constructed for each pair of classes, and the examples belonging to the other classes are discarded. Figures 1 and 2 schematically illustrate these approaches. For a $M$-class problem ( $M$ music genres) several classification results arise: according to the OAA technique $M$ class labels are produced, whereas for the RR technique $M(M-1) / 2$ class labels are generated. These labels are combined according to a decision procedure in order to produce the final class label.

We emphasize that the decomposition here is made only by manipulating the instances in the considered database: relabeling conveniently the negative examples in the OAA approach, and discarding the examples of the non-considered classes in the RR approach. Hence, each one of the generated classification problem follows exactly the formal description presented in the previous section. 


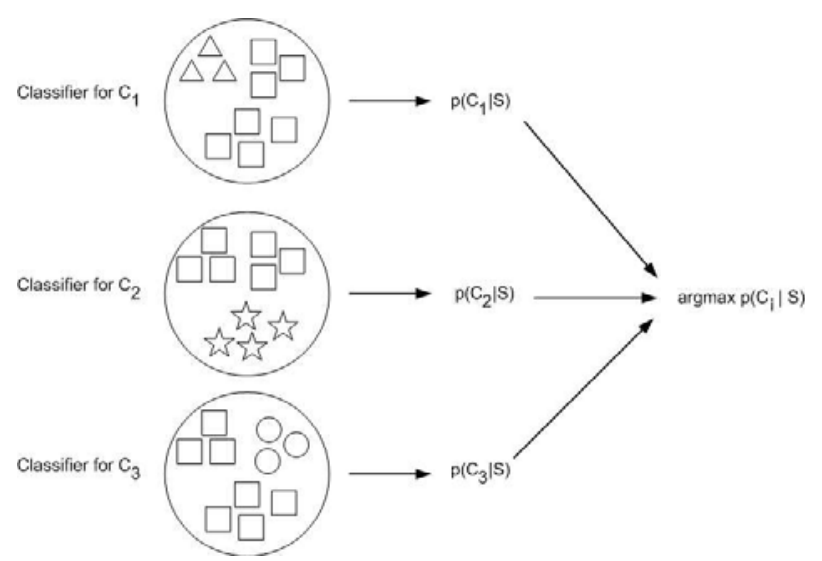

Figure 1. One-Against-All Space Decomposition Approach

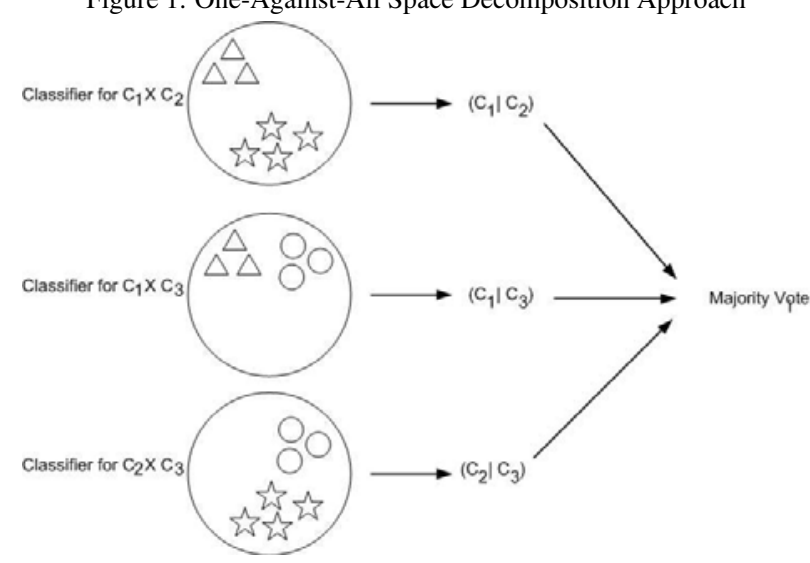

Figure 2. Round-Robin Space Decomposition Approach

\subsection{TIME DECOMPOSITION}

An audio record of a music piece is a time-varying signal. The idea behind time decomposition is that we can obtain a more adequate representation of the music piece if we consider several time segments of the signal. This procedure aims to better treat the great variation that usually occurs along music pieces, and also permits to compare the discriminative power of the features extracted from different parts of the music.

Figure 3 illustrates this point: it presents the average values of 30 features extracted from different music sub-intervals, obtained over 150 music pieces of the genre Salsa. We emphasize the irregularity of the results, showing that feature values vary depending on the interval from which they were obtained.

If we employ the formal description of the AMGC problem given in the previous section, the time decomposition can be formalized as follows. From the original music signal $S=<s_{1}, s_{2} \ldots s_{N}>$ we obtain different subsignals $S_{p q}$. Each sub-signal is simply a projection of $S$ on the interval $[p, q]$ of samples, or $S_{p q}=<s_{p}, \ldots s_{q}>$. In the generic case that uses $K$ sub-signals, we further

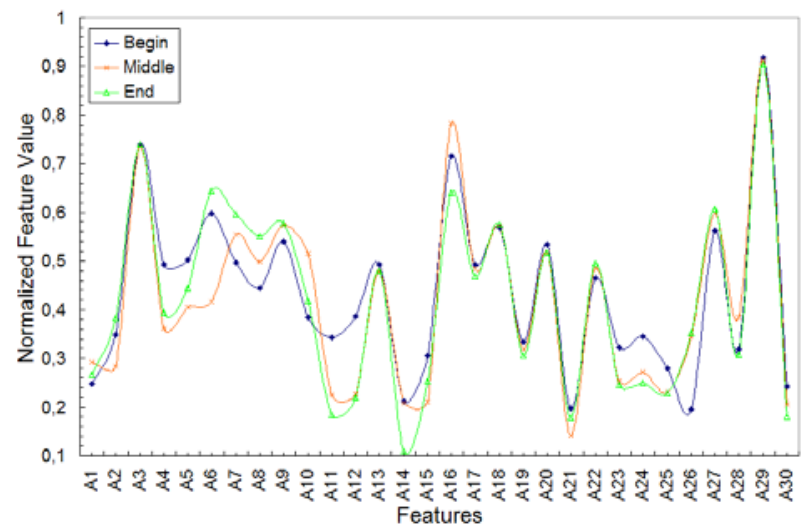

Figure 3. Average values over 150 music pieces of genre Salsa for 30 features extracted from different music sub-intervals

obtain a sequence of feature vectors $\bar{X}_{1}, \bar{X}_{2} \ldots \bar{X}_{K}$. A classifier is applied in each one of these feature vectors, generating the assigned music genres $\hat{g}_{1}, \hat{g}_{2} \ldots \hat{g}_{K}$; then they must be combined to produce the final class assignment, as we will see in the following.

In our case we employ feature vectors extracted from 30 -seconds segments from the beginning $\left(S_{b e g}\right)$, middle $\left(S_{m i d}\right)$ and end $\left(S_{\text {end }}\right)$ parts of the original music signal. The corresponding feature vectors are denoted $\bar{X}_{b e g}$, $\bar{X}_{m i d}$ and $\bar{X}_{\text {end }}$. Figure 4 illustrates the time decomposition process.

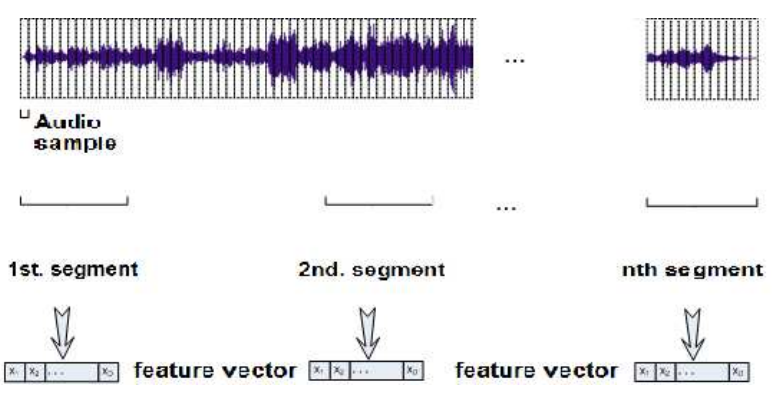

Figure 4. Time Decomposition Approach

\subsection{THE SET OF EMPLOYED FEATURES}

The MARSYAS framework was employed for feature extraction, so we use the same feature set proposed by Tzanetakis and Cook [35]. These features can grouped in three categories: Beat-Related, Timbral Texture and Pitch Related. The Beat-Related features (features 1 to 6) include the relative amplitudes and the beat per minute. The Timbral Texture features (features 7 to 25) account for the means and variance of the spectral centroid, rolloff, flux, the time zero domain crossings, the first 5 Mel Frequency Cepstral Coefficients (MFCC) and low energy. PitchRelated features (features 26 to 30) include de maximum periods of the pitch peak in the pitch histograms. The final feature vector is 30-dimensional (Beat: 6; Timbral 
Texture: 19; Pitch: 5). For a more detailed description of the features refer to [34] or [35] .

A normalization procedure is applied, in order to homogenize the input data for the classifiers: if $\max V$ and $\min V$ are the maximum and minimum values that appear in all dataset for a given feature, a value $V$ is replaced by $n e w V$ using the equation

$$
n e w V=\frac{(V-\min V)}{(\max V-\min V)}
$$

The final feature vector is outlined in Table 1.

Table 1. Feature vector description

\begin{tabular}{|c|l|}
\hline Feature \# & Description \\
\hline 1 & Relative amplitude of the first histogram peak \\
\hline 2 & Relative amplitude of the second histogram peak \\
\hline 3 & $\begin{array}{l}\text { Ratio between the amplitudes of the second peak } \\
\text { and the first peak }\end{array}$ \\
\hline 4 & Period of the first peak in bpm \\
\hline 5 & Period of the second peak in bpm \\
\hline 6 & Overall histogram sum (beat strength) \\
\hline 7 & Spectral centroid mean \\
\hline 8 & Spectral rolloff mean \\
\hline 9 & Spectral flow mean \\
\hline 10 & Zero crossing rate mean \\
\hline 11 & Standard deviation for spectral centroid \\
\hline 12 & Standard deviation for spectral rolloff \\
\hline 13 & Standard deviation for spectral flow \\
\hline 14 & Standard deviation for zero crossing rate \\
\hline 15 & Low energy \\
\hline 16 & 1 st. MFCC mean \\
\hline 17 & 2 nd. MFCC mean \\
\hline 18 & 3 rd. MFCC mean \\
\hline 19 & 4 th. MFCC mean \\
\hline 20 & 5 th. MFCC mean \\
\hline 21 & Standard deviation for 1 st. MFCC \\
\hline 22 & Standard deviation for 2 nd. MFCC \\
\hline 23 & Standard deviation for 3 rd. MFCC \\
\hline 24 & Standard deviation for 4 th. MFCC \\
\hline 25 & Standard deviation for 5 th. MFCC \\
\hline 26 & The overall sum of the histogram (pitch strength) \\
\hline 27 & $\begin{array}{l}\text { Period of the maximum peak of the } \\
\text { unfolded histogram }\end{array}$ \\
\hline 28 & $\begin{array}{l}\text { Amplitude of maximum peak of the } \\
\text { folded histogram }\end{array}$ \\
\hline 29 & $\begin{array}{l}\text { Period of the maximum peak of the } \\
\text { folded histogram }\end{array}$ \\
\hline 30 & $\begin{array}{l}\text { Pitch interval between the two most prominent } \\
\text { peaks of the folded histogram }\end{array}$ \\
\hline & \\
\hline 2 & \\
\hline 2 & \\
\hline 2 &
\end{tabular}

We note that the feature vectors are always calculated over intervals; in fact several features like means, variances, and number of peaks, only have meaning if extracted from signal intervals. So they are calculated over $S$ or one of its subintervals $S_{p q}$, that is, an aggregate segment obtained from the elementary frames of the music audio signal.

\subsection{Classification and COMbination DECI- SION PROCEDURES}

A large set of standard algorithms for supervised machine learning is used to accomplish the AMGC task. We follow an homogeneous approach, that is, the very same classifier is employed as individual component classifier in each music part. We use the following algorithms [28]: (a) a classic decision tree classifier (J48); (b) the instance-based $k$-NN classifier; (c) the Naïve-Bayes classifier (NB), which is based on conditional probabilities and attribute independence; (d) a Multi Layer Perceptron neural network (MLP) with the backpropagation momentum algorithm; and (e) a Support Vector Machine classifier (SVM) with pairwise classification. In order to do all experiments we employ a framework based on the WEKA Datamining Tool [37], with standard parameters.

As previously mentioned, a set of possibly different candidate classes is produced by the individual classifiers. These results can be considered according to space and time decomposition dimensions. The time dimension presents $K$ classification results, and space dimension for a $M$-class problem produces $M(\mathrm{OAA})$ or $M(M-1) / 2$ (RR) results, as already explained. These partial results must be composed to produce the final class label.

We employ a decision procedure in order to find the final class associated by the ensemble of classifiers. Space decomposition results are combined by the majority vote rule, in the case of the RR approach, and by a rule based on the a posteriori probability of the employed classifier, for the OAA approach. Time decomposition results are combined using the majority vote rule.

\section{SPACE-TIME DECOMPOSITION EX- PERIMENTS AND RESULTS}

One common concern in the AMGC problem research is how reliable the obtained experiments and results are, because the way the musical genres are associated to the music pieces in the employed databases. Craft, Wiggins and Crawford [5] raise this question and argue that genre labeling is affected by two major factors: the first one is related to how the composer intended to draw upon different stylistic elements from one or more music genres; the second one is related to the social and cultural background of any participant involved in labeling the database. They conclude that the evaluation of a genre labeling system is highly dependent upon these two factors, and recommend that for the evaluation of genre labeling systems which use individual genres these cultural differences should be eliminated. However, according to McEnnis and Cunningham [26] cultural differences - or social context should be preserved, because they play an important role in the human subjectiveness associated to the task of assigning musical genres to music pieces. In the work of 
Table 2. Accuracy (\%) using OAA and RR approaches in the individual segments

\begin{tabular}{|c|c|c|c|c|c|c|c|c|c|}
\hline & \multicolumn{3}{|c|}{$S_{\text {beg }}$} & \multicolumn{3}{c|}{$S_{\text {mid }}$} & \multicolumn{3}{c|}{$S_{\text {end }}$} \\
\hline Classifier & BL & OAA & RR & BL & OAA & RR & BL & OAA & RR \\
\hline J48 & 39.60 & 41.56 & 45.96 & 44.44 & 44.56 & $\mathbf{4 9 . 9 3}$ & 38.80 & 38.42 & 45.53 \\
\hline 3-NN & 45.83 & 45.83 & 45.83 & $\mathbf{5 6 . 2 6}$ & $\mathbf{5 6 . 2 6}$ & $\mathbf{5 6 . 2 6}$ & 48.43 & 48.43 & 48.43 \\
\hline MLP & 53.96 & 52.53 & 55.06 & $\mathbf{5 6 . 4 0}$ & 53.08 & 54.59 & 48.26 & 51.96 & 51.92 \\
\hline NB & 44.43 & 42.76 & 44.43 & 47.76 & 45.83 & $\mathbf{4 7 . 7 9}$ & 39.13 & 37.26 & 39.19 \\
\hline SVM & - & 26.63 & 57.43 & - & 36.82 & $\mathbf{6 3 . 5 0}$ & - & 28.89 & 54.60 \\
\hline
\end{tabular}

McEnnis, McKay and Fujinaga [25] the issue of properly constructing databases that can be useful for other research works is addressed.

Considering these concerns of the research community, and in order to accomplish the desired AMGC task, a new database was constructed: the Latin Music Database (feature vectors available in www.ppgia.pucpr.br/ silla/lmd/) [31], [32] [33], [34]. This database contains 3,160 MP3 music pieces of 10 different Latin genres, originated from music pieces of 543 artists.

In this database music genre assignment was manually made by a group of human experts, based on the human perception of how each music is danced. The genre labeling was performed by two professional teachers with over 10 years of experience in teaching ballroom Latin and Brazilian dances. The professionals made a first selection of the music they considered to be relevant to a specific genre regarding the way it is danced; the project team did a second verification in order to avoid mistakes. The professionals classified around 300 music pieces per month, and the development of the complete database took around one year.

In order to verify the application of our proposal in the AMGC problem an extensive set of tests were conducted. We consider two main goals: (a) to verify if feature vectors extracted from different parts of the audio signal have similar discriminant power in the AMGC task; and (b) to verify if the proposed ensemble approach, encompassing space and time decompositions, provides better results than classifiers applied to a single feature vector extracted from the music audio signal. Our primary evaluation measure is the classification accuracy, that is, the average number of correctly classified music pieces.

The experiments were carried out on stratified training, validation and test datasets. In order to deal with balanced classes, 300 different song tracks from each genre were randomly selected. In the experiments we use a tenfold cross-validation procedure, that is, the presented results are obtained from 10 randomly independent experiment repetitions.

For time decomposition we use three time segments of 30 seconds each, extracted from the beginning, middle and end parts of each music piece. We note that 30 -seconds are equivalent to 1,153 frames in a MP3 file. According to the explained formalism we have, for a music signal composed of $N$ samples: $S_{b e g}=<s_{1}, \ldots s_{1,153}>$, $S_{\text {mid }}=<s_{\left(\frac{N}{3}\right)+500}, \ldots s_{\left(\frac{N}{3}\right)+1,653}>\quad$ and $\left.S_{\text {end }}=<s_{N-1,153-300}, \ldots s_{N-300}\right\rangle . \quad$ An empiric displacement of 300 sample frames was employed in order to discard the final part of the music, which is usually constituted by silence or noise.

Table 2 presents the results for each classifier in the segments $S_{\text {beg }}, S_{m i d}$ and $S_{\text {end }}$ for the OAA and RR approaches. Column BL stands for baseline, and shows the results for the indicated classifier without space decomposition. As the SVM classifier was employed as default for the RR approach, in this case the results for the BL column were omitted.

The following analysis can be done on the results shown in Table 2: (a) for the J48 classifier the RR approach increases accuracy, but for the OAA approach the increment is not significative; (b) the 3-NN classifier presents the same results for every segment, but they vary according to the adopted strategy; (c) for the MLP classifier the decomposition strategies increase accuracy in the beginning segment for RR, and in the end segment for OAA and RR; (d) for the NB classifier the approaches did not increase significantly the classification performance; and (e) the SVM classifier presents not only the best classification results, when using RR, but also the worst one in every segment when employing the OAA approach. A global view shows that the RR approach surpasses the OAA approach for most classifiers; the only exception is the case MLP for $S_{m i d}$.

Table 3 presents the results for the space-time ensemble decomposition strategy in comparison with the space decomposition strategy applied to the entire music piece. In this table the TD (Time Decomposition) column indicate values obtained without space decomposition, but using time decomposition with the majority vote rule. The column BL (Baseline) stands for the application of the classifier in the entire music piece with no decompositions.

Results in Table 3 show that for the entire music piece the use of the RR strategy increases classification accuracy of any classifier regardless the baseline, whereas the 
Table 3. Accuracy (\%) using space-time decomposition versus entire music piece

\begin{tabular}{|c|c|c|c|c|c|c|}
\hline & \multicolumn{3}{|c|}{ Space-time Ensembles } & \multicolumn{3}{c|}{ Entire Music } \\
\hline Classifier & TD & OAA & RR & BL & OAA & RR \\
\hline J48 & 47.33 & 49.63 & $\mathbf{5 4 . 0 6}$ & 44.20 & 43.79 & 50.63 \\
\hline 3-NN & 60.46 & 59.96 & $\mathbf{6 1 . 1 2}$ & 57.96 & 57.96 & 59.93 \\
\hline MLP & 59.43 & $\mathbf{6 1 . 0 3}$ & 59.79 & 56.46 & 58.76 & 57.86 \\
\hline NB & 46.03 & 43.43 & 47.19 & 48.00 & 45.96 & $\mathbf{4 8 . 1 6}$ \\
\hline SVM & - & 30.79 & $\mathbf{6 5 . 0 6}$ & - & 37.46 & 63.40 \\
\hline
\end{tabular}

OAA strategy presents superior results only for the MLP neural net classifier. When comparing the global results for the entire music piece, the RR strategy results overcome the OAA strategy in most cases.

In the case of using combined space-time decomposition, both OAA and RR strategies marginally increase classification accuracy. When comparing the entire music with space-time decomposition the results are similar of the ones in the previous experiments: for $\mathrm{J} 48,3-\mathrm{NN}$ and MLP in all cases the decomposition results are better; for NB the results are inconclusive; and for SVM the results are superior only for the RR strategy. The best overall result is achieved using SVM with space-time decomposition and the RR approach.

\section{Feature Selection AND RElated EXPERIMENTS}

The feature selection (FS) task is the selection of a proper subset of original feature set, in order to simplify and reduce the preprocessing and classification steps, but assuring the same or upper final classification accuracy [3], [6].

The feature selection methods are often classified in two groups: the filter approach and the wrapper approach [29]. In the filter approach the feature selection process is carried out before the use of any recognition algorithm, as a preprocessing step. In the wrapper approach the pattern recognition algorithm is used as a sub-routine of the system to evaluate the generated solutions.

In our system we employ several feature vectors, according to space and time decompositions. The feature selection procedure is employed in the different time segment vectors, allowing us to compare the relative importance and/or discriminative power of each feature according to their time origin. Another goal is to verify how the results obtained with the ensemble-based method are affected by the features selected from the component segments.

The employed feature selection procedure is based on the genetic algorithm (GA) paradigm and uses the wrapper approach. Individuals - chromosomes in the GA paradigm - are $F$-dimensional binary vectors, where
$F$ is the maximum feature vector size; in our case we have $F=30$, the number of features extracted by the MARSYAS framework.

The GA general procedure can be summarized as follows [34]:

1. each individual works as a binary mask for the associated feature vector;

2. an initial assignment is randomly generated: a value 1 indicates that the corresponding feature must be used, and 0 that it must be discarded;

3. a classifier is trained using only the selected features;

4. the generated classification structure is applied to a validation set to determine the fitness value of this individual;

5. we proceed elitism to conserve the best individuals; crossover and mutation operators are applied in order to obtain the next generation; and

6. steps 3 to 5 are repeated until the stopping criteria is attained.

In our feature selection procedure each generation is composed of 50 individuals, and the evolution process ends when it converges - no significant change occurs in successive generations - or when a fixed max number of generations is achieved.

Tables 4, 5 and 6 present the results obtained with the feature selection procedure applied to the beginning, middle and end music segments, respectively [34]. In these tables the classifier is indicated in the first column; the second column presents a baseline (BL) result, which is obtained applying the corresponding classifier directly to the complete feature vector obtained from the MARSYAS framework; columns 3 and 4 show the results for OAA and RR space decomposition approaches without feature selection; columns FS, FSOAA and FSRR show the corresponding results with the feature selection procedure.

Table 4. Classification accuracy (\%) using space decomposition for the beginning segment of the music $\left(S_{b e g}\right)$

\begin{tabular}{|c|c|c|c|c|c|c|}
\hline Classifier & BL & OAA & RR & FS & FSOAA & FSRR \\
\hline J48 & 39.60 & 41.56 & 45.96 & 44.70 & 43.52 & $\mathbf{4 8 . 5 3}$ \\
\hline 3-NN & 45.83 & 45.83 & 45.83 & 51.19 & 51.73 & $\mathbf{5 3 . 3 6}$ \\
\hline MLP & 53.96 & 52.53 & $\mathbf{5 5 . 0 6}$ & 52.73 & 53.99 & 54.13 \\
\hline NB & 44.43 & 42.76 & 44.43 & $\mathbf{4 5 . 4 3}$ & 43.46 & 45.39 \\
\hline SVM & - & 23.63 & $\mathbf{5 7 . 4 3}$ & - & 26.16 & 57.13 \\
\hline
\end{tabular}

Analyzing these results for each classifier we can outline the following conclusions: (a) for J48 and 3-NN the feature selection method with the RR space-time decomposition approach produces better accuracy than the other 
Table 5. Classification accuracy (\%) using space decomposition for the middle segment of the music $\left(S_{\text {mid }}\right)$

\begin{tabular}{|c|c|c|c|c|c|c|}
\hline Classifier & BL & OAA & RR & FS & FSOAA & FSRR \\
\hline J48 & 44.44 & 44.56 & 49.93 & 45.76 & 45.09 & $\mathbf{5 0 . 8 6}$ \\
\hline 3-NN & 56.26 & 56.26 & 56.26 & 60.02 & 60.95 & $\mathbf{6 2 . 5 5}$ \\
\hline MLP & $\mathbf{5 6 . 4 0}$ & 53.08 & 54.59 & 54.73 & 54.76 & 49.76 \\
\hline NB & 47.76 & 45.83 & 47.79 & 50.09 & 48.79 & $\mathbf{5 0 . 6 9}$ \\
\hline SVM & - & 38.62 & $\mathbf{6 3 . 5 0}$ & - & 32.86 & 59.70 \\
\hline
\end{tabular}

Table 6. Classification accuracy (\%) using space decomposition for the end segment of the music $\left(S_{\text {end }}\right)$

\begin{tabular}{|c|c|c|c|c|c|c|}
\hline Classifier & BL & OAA & RR & FS & FSOAA & FSRR \\
\hline J48 & 38.80 & 38.42 & 45.53 & 38.73 & 38.99 & $\mathbf{4 5 . 8 6}$ \\
\hline 3-NN & 48.43 & 48.43 & 48.43 & 51.11 & 51.10 & $\mathbf{5 3 . 4 9}$ \\
\hline MLP & 48.26 & $\mathbf{5 1 . 9 6}$ & 51.92 & 47.86 & 50.53 & 49.64 \\
\hline NB & 39.13 & 37.26 & 39.19 & $\mathbf{3 9 . 6 6}$ & 37.63 & 39.59 \\
\hline SVM & - & 28.89 & 54.60 & - & 28.22 & $\mathbf{5 5 . 3 3}$ \\
\hline
\end{tabular}

options; (b) for the MLP classifier feature selection seems to be ineffective: best results are obtained with the complete feature set; (c) for the NB classifier the FS produces the better results without space decomposition in $S_{b e g}$ and $S_{\text {end }}$, and with the RR approach in $S_{m i d}$; (d) for the SVM classifier the best results arrive with the use of the RR approach, and FS increase accuracy only in the $S_{\text {end }}$ segment. This classifier also presents the best overall result: using the RR space decomposition in $S_{m i d}$ without feature selection.

In order to consider the ensemble approach with time decomposition, Table 7 presents the results of the conducted experiments using space and time decompositions, for OAA and RR approaches, with and without feature selection. We emphasize that this table encompasses three time segments $S_{b e g}, S_{m i d}$ and $S_{\text {end }}$, merged according to the already described combination procedure.

Table 7. Classification accuracy (\%) using global space-time decompositions

\begin{tabular}{|c|c|c|c|c|c|c|}
\hline Classifer & BL & OAA & RR & FS & FSOAA & FSRR \\
\hline J48 & 47.33 & 49.63 & 54.06 & 50.10 & 50.03 & $\mathbf{5 5 . 4 6}$ \\
\hline 3-NN & 60.46 & 59.96 & 61.12 & 63.20 & 62.77 & $\mathbf{6 4 . 1 0}$ \\
\hline MLP & 59.43 & $\mathbf{6 1 . 0 3}$ & 59.79 & 59.30 & 60.96 & 56.86 \\
\hline NB & 46.03 & 43.43 & 47.19 & 47.10 & 44.96 & $\mathbf{4 9 . 7 9}$ \\
\hline SVM & - & 30.79 & $\mathbf{6 5 . 0 6}$ & - & 29.47 & 63.03 \\
\hline
\end{tabular}

Summarizing the results in Table 7, we conclude that the FSRR method improves classification accuracy for the classifiers J48, 3-NN and NB. Also, OAA and FSOAA methods present similar results for the MLP classifier, and only for the SVM classifier the best result is obtained without FS.

These results - and also the previous ones obtained in the individual segments - indicate that space decomposition and feature selection are more effective for classifiers that produce simple separation surfaces between classes, like J48, 3-NN and NB, in contrast with the results obtained for the MLP and SVM classifiers, which can produce complex separation surfaces. This situation corroborates our initial hypothesis related to the use of space decomposition strategies.

As already mentioned, we also want to analyze if different features have the same importance according to their time origin. Table 8 shows a schematic map indicating the features selected in each time segment by our FS procedure. In this table we employ a binary BME mask - for (B)eginning, (M)iddle and (E)nd time segments where 1 indicates that the feature was selected in the corresponding time segment, and 0 otherwise.

Table 8. Selected features in each time segment (BME mask)

\begin{tabular}{|c|c|c|c|c|c|c|}
\hline Feature & 3-NN & $\mathrm{J} 48$ & MLP & NB & SVM & $\#$ \\
\hline 1 & 000 & 001 & 010 & 101 & 111 & 7 \\
\hline 2 & 000 & 000 & 010 & 010 & 011 & 4 \\
\hline 3 & 000 & 001 & 010 & 011 & 000 & 4 \\
\hline 4 & 000 & 111 & 010 & 111 & 001 & 8 \\
\hline 5 & 000 & 000 & 110 & 101 & 100 & 5 \\
\hline 6 & 111 & 101 & 111 & 111 & 110 & 13 \\
\hline 7 & 011 & 110 & 110 & 000 & 100 & 7 \\
\hline 8 & 001 & 111 & 110 & 000 & 111 & 9 \\
\hline 9 & 111 & 111 & 111 & 111 & 111 & 15 \\
\hline 10 & 110 & 011 & 111 & 111 & 111 & 13 \\
\hline 11 & 100 & 001 & 111 & 001 & 110 & 8 \\
\hline 12 & 011 & 010 & 111 & 011 & 111 & 11 \\
\hline 13 & 111 & 011 & 111 & 111 & 111 & 14 \\
\hline 14 & 001 & 010 & 101 & 000 & 011 & 6 \\
\hline 15 & 011 & 111 & 111 & 111 & 111 & 14 \\
\hline 16 & 111 & 111 & 111 & 111 & 111 & 15 \\
\hline 17 & 111 & 100 & 111 & 111 & 111 & 13 \\
\hline 18 & 111 & 111 & 111 & 111 & 111 & 15 \\
\hline 19 & 111 & 010 & 111 & 111 & 111 & 13 \\
\hline 20 & 011 & 010 & 110 & 101 & 101 & 9 \\
\hline 21 & 111 & 111 & 111 & 101 & 111 & 14 \\
\hline 22 & 111 & 110 & 111 & 111 & 111 & 14 \\
\hline 23 & 111 & 111 & 111 & 100 & 111 & 13 \\
\hline 24 & 011 & 000 & 111 & 001 & 011 & 8 \\
\hline 25 & 111 & 011 & 101 & 111 & 111 & 13 \\
\hline 26 & 000 & 010 & 100 & 111 & 111 & 8 \\
\hline 27 & 000 & 111 & 000 & 101 & 101 & 7 \\
\hline 28 & 111 & 111 & 011 & 111 & 111 & 14 \\
\hline 29 & 000 & 100 & 000 & 000 & 101 & 3 \\
\hline 30 & 000 & 011 & 000 & 111 & 000 & 5 \\
\hline
\end{tabular}

Several conclusions can be draw from Table 8. The last column in this table indicates how many times the corresponding feature was selected in the experiments (max 15 selections). Although different features can have different importance according to the classifier, we argue that this counting gives a global idea of the discrimina- 
tive power of each feature. For example, features $6,9,10$, $13,15,16,17,18,13,21,22,23,25$ and 28 are highly selected, so they are important for music genre classification. For more discussion, see [31], [33] and [34]. We remember that features 1 to 6 are Beat related, 7 to 25 are related to Timbral Texture, and 26 to 30 are Pitch related.

\section{Conclusions}

In this paper we present a novel approach to the Music Genre Classification Problem, which is based on ensemble approach and the decomposition of the music signal according to space and time dimensions. Feature vectors are selected from different time segments of the beginning, middle and end parts of the music; in order to apply simple but effective classifiers, space decomposition strategies based on the One-Against-All and RoundRobin approaches were used. From the set of partial classification results originated from these views of the problem space, an unique final classification label is provided. A large brand of classical categorization algorithms were employed in the individual segments, and an heuristic combination procedure was used to produce the final music genre label.

In order to evaluate the proposal we have conducted a extensive set of experiments in a relatively large database - the Latin Music Database, with more than 3,000 music pieces from 10 music genres - specially constructed for this research project. This database was methodically constructed and is open to new research projects in the area.

Several conclusions can be inferred from the obtained results. Firstly, we conclude that the use of the initial 30 -second segment of the beginning of the music piece - which is the most frequent strategy used up to now to obtain the music feature vector - is not adequate: our test results show that the middle part is better than the initial or the end parts of the music signal for feature extraction (Table 2). We believe that this phenomena occurs because this middle part the music signal is more stable and more compatible with the corresponding music genre than the others. In fact, results obtained using the middle segment are similar to the ones using the complete music signal; in the latter case, however, the processing time is higher, since there is an obvious relation between the length of the time interval used for feature extraction and the computational complexity of the corresponding extraction procedure.

Secondly, we conclude that the use of three time segments and the ensemble of classifiers approach provide better results in accuracy for the AMGC task than the ones obtained from the individual segments (Table 3). This result is in accordance with the conclusions of Li, Ogihara and $\mathrm{Li}$ [21], who state that specific approaches must be used for labeling different music genres when some hierarchical classification is considered. So, we believe that our space-time decomposition scheme provides better classification results. Unfortunately a direct comparison with the results of Tzanetakis and Cook [35], or the ones of Li, Ogihara and $\mathrm{Li}$ [21] is not possible because the GTZAN database provides only the feature values for the initial 30-second segment of the music pieces. Our temporal approach also differs from the one employed by Bergstra et al. [2], that initially uses simple decision stumps applied individually to each feature, and then feature selection and classification in parallel using AdaBoost.

In third place we can analyze the results concerning the space decomposition approaches. As already mentioned, the use of a set of binary classifiers is adequate in problems that present complex class separation surfaces. In general our results show that the RR approach presents superior results regarding the OAA approach (Tables 2 and 3). We justify this fact using the same explanation: in $\mathrm{RR}$ individual instances are eliminated - in comparison with the relabeling in the OAA approach - so the construction of the separation surface by the classification algorithm is simplified. Our best classification accuracy result was obtained with the SVM classifier and space-time decomposition according to the RR approach.

We also evaluate the effect of using a feature selection procedure in the AMGC problem. Our FS procedure is based on the genetic algorithm paradigm. Each individual works as a mask that selects the set of features to be used for classification. The fitness of the individuals is based on the classification performance according to the wrapper approach. Classical genetic operations (crossover, mutation, elitism) are applied until a stopping criteria is attained.

The results achieved with FS show that this procedure is effective for J48, $k$-NN and Naïve-Bayes classifiers; for MLP and SVM the FS procedure does not increases classification accuracy (Tables 4, 5, 6 and 7); these results are compatible with the ones presented in [38]. We note that using a reduced set of features implies a smaller processing time; this is an important issue in practical applications, where a compromise between accuracy and efficiency must be achieved.

We also note that the features have different importance in the classification, according to their music segment origin (Table 8). It can be seen, however, that some features are present in almost every selection, showing that they have a strong discriminative power in the classification task.

In summary, the use of space-time decomposition and the ensemble of classifiers approach provide better accuracy for music genre labeling than the use of individual segments - initial, middle and end parts - of the music signal, even when the classifier is trained with the whole 
music signal. Also, our approach represents an interesting trade-off between computational effort and classification accuracy, an important issue in practical applications. Indeed, the origin, number and duration of the time segments, the set of discriminative features, and the use of an adequate space decomposition strategy still remain open questions for the AMGC problem.

We intend to improve our proposal in order to increase classification accuracy, by adding a second layer of binary classifiers to deal with classes and/or partial state space views that present higher confusion.

\section{REFERENCES}

[1] J.J. Aucouturier; F. Pachet. Representing musical genre: a state of the art. Journal of New Music Research, 32(1):83-93, 2003.

[2] J. Bergstra; N. Casagrande; D. Erhan; D. Eck; B. Kégl. Aggregate features and ADABOOST for music classification. Machine Learning, 65(2-3):473484, 2006

[3] A. Blum; P. Langley. Selection of relevant features and examples in Machine Learning. Artificial Intelligence, 97(1-2):245-271, 1997.

[4] C.H.L. Costa; J. D. Valle Jr; A.L. Koerich. Automatic classification of audio data. IEEE International Conference on Systems, Man, and Cybernetics, pages 562-567, 2004.

[5] A.J.D. Craft; G.A. Wiggins; T. Crawford. How many beans make five? the consensus problem in Music Genre Classification and a new evaluation method for single genre categorization systems. Proceedings of the 8th International Conference on Music Information Retrieval, Vienna, Austria, pages 73-76, 2007.

[6] M. Dash; H. Liu. Feature selection for classification. Intelligent Data Analysis, 1(1-4):131-156, 1997.

[7] T.G. Dietterich. Ensemble methods in Machine Learning. Proceedings of the 1st. International Workshop on Multiple Classifier System, Lecture Notes in Computer Science, 1857:1-15, 2000.

[8] J.S. Downie; S.J. Cunningham. Toward a theory of music information retrieval queries: system design implications. Proceedings of the 3rd International Conference on Music Information Retrieval, pages 299-300, 2002.
[9] J.S. Downie. The Music Information Retrieval Evaluation eXchange (MIREX). D-Lib Magazine, 12(12), 2006.

[10] R. Fiebrink; I. Fujinaga. Feature selection pitfalls and music classification. Proceedings of the 7th International Conference on Music Information Retrieval, Victoria, Canada, pages 340-341, 2006.

[11] Y. Freund; R. E. Schapire. A decision-theoretic generalization of on-line learning and an application to boosting. Journal of Computer and System Sciences, 55(1):119-139, 1997.

[12] J. Fürnkranz. Pairwise Classification as an ensemble technique. Proceedings of the 13th European Conference on Machine Learning, Helsinki, Finland, pages 97-110, 2002.

[13] M. Grimaldi; P. Cunningham; A. Kokaram. A wavelet packet representation of audio signals for music genre classification using different ensemble and feature selection techniques. Proceedings of the 5th ACM SIGMM International Workshop on Multimedia Information Retrieval, ACM Press, pages 102-108, 2003.

[14] M. Grimaldi; P. Cunningham; A. Kokaram. An evaluation of alternative feature selection strategies and ensemble techniques for classifying music. Workshop on Multimedia Discovery and Mining, 14th European Conference on Machine Learning, 7th European Conference on Principles and Practice of Knowledge Discovery in Databases, Dubrovnik, Croatia, 2003.

[15] S. Hacker. MP3: The Definitive Guide. O'Reilly Publishers, 2000

[16] T.K. Ho. Nearest neighbors in random subspaces. Advances in Pattern Recognition, Joint IAPR International Workshops SSPR and SPR, Lecture Notes in Computer Science, 1451:640-648, 1998.

[17] J. Kittler; M. Hatef; R.P.W. Duin; and J. Matas. On Combining Classifiers. IEEE Transactions on Pattern Analysis and Machine Intelligence, 20(3):226239, 1998.

[18] A.L. Koerich; C. Poitevin. Combination of homogenuous classifiers for musical genre classification. IEEE International Conference on Systems, Man and Cybernetics, IEEE Press, Hawaii, USA, pages 554-559, 2005.

[19] K. Kosina. Music Genre Recognition. MSc. Dissertation, Fachschule Hagenberg, June 2002. 
[20] J.H. Lee; J.S. Downie. Survey of music information needs, uses, and seeking behaviours preliminary findings. Proceedings of the 5th International Conference on Music Information Retrieval, Barcelona, Spain, pages 441-446, 2004.

[21] T. Li; M. Ogihara; Q. Li. A Comparative study on content-based Music Genre Classification. Proceedings of the 26th Annual International ACM SIGIR Conference on Research and Development in Informaion Retrieval, Toronto, ACM Press, pages 282-289, 2003.

[22] M. Li; R. Sleep. Genre classification via an LZ78based string kernel. Proceedings of the 6th International Conference on Music Information Retrieval, London, United Kingdom, pages 252-259, 2005.

[23] T. Li; M. Ogihara. Music Genre Classification with taxonomy. Proceedings of IEEE International Conference on Acoustics, Speech and Signal Processing, Philadelphia, USA, pages 197-200, 2005.

[24] H. Liu; L. Yu. Feature extraction, selection, and construction. The Handbook of Data Mining, Lawrence Erlbaum Publishers, chapter 16, pages 409-424, 2003.

[25] D. McEnnis; C. McKay; I. Fujinaga. Overview of OMEN (On-demand Metadata Extraction Network). Proceedings of the International Conference on Music Information Retrieval, Victoria, Canada, pages 7-12, 2006.

[26] D. McEnnis; S.J. Cunningham. Sociology and music recommendation systems. Proceedings of the 8th International Conference on Music Information Retrieval, Vienna, Austria, pages 185-186, 2007.

[27] A. Meng; P. Ahrendt; J. Larsen. Improving Music Genre Classification by short-time feature integration. Proceedings of the IEEE International Conference on Acoustics, Speech, and Signal Processing, Philadelphia, USA, pages 497-500, 2005.

[28] T. M. Mitchell. Machine Learning. McGraw-Hill, 1997.

[29] L.C. Molina; L. Belanche; A. Nebot. Feature selection algorithms: a survey and experimental evaluation. Proceedings of the IEEE International Conference on Data Mining, Maebashi City, Japan, pages 306-313, 2002.

[30] E. Pampalk; A. Rauber; D. Merkl. Content-Based organization and visualization of music archives. Proceedings of ACM Multimedia, Juan-les-Pins, France, pages 570-579, 2002.
[31] C.N. Silla Jr.; C.A.A. Kaestner; A. L. Koerich. Time-Space ensemble strategies for automatic music genre classification. Proceedings of the 18th Brazilian Symposium on Artificial Intelligence, Ribeiro Preto, Brazil, Lecture Notes in Computer Science, 4140:339-348, 2006.

[32] C.N. Silla Jr.; C.A.A. Kaestner; A. L. Koerich. The Latin Music Database: a database for the automatic classification of music genres (in portuguese). Proceedings of 11th Brazilian Symposium on Computer Music, So Paulo, BR, pages 167-174, 2007.

[33] C.N. Silla Jr.; C.A.A. Kaestner; A.L. Koerich. Automatic music genre classification using ensemble of classifiers. Proceedings of the IEEE International Conference on Systems, Man and Cybernetics, Montreal, Canada, pages 1687-1692, 2007.

[34] C. N. Silla Jr.. Classifiers Combination for Automatic Music Classification (in portuguese). MSc. Dissertation, Graduate Program in Applied Computer Science, Pontifical Catholic University of Paraná, January 2007.

[35] G. Tzanetakis; P. Cook. Musical genre classification of audio signals. IEEE Transactions on Speech and Audio Processing, 10(5):293-302, 2002.

[36] F. Vignoli. Digital music interaction concepts: a user study. Proceedings of the 5th International Conference on Music Information Retrieval, Barcelona, Spain, pages 415-420, 2004.

[37] I. H. Witten; E. Frank. Data Mining: Practical Machine Learning Tools and Techniques. Morgan Kaufmann, 2005.

[38] Y. Yaslan; Z. Cataltepe. Audio music genre classification using different classifiers and feature selection methods. Proceedings of the International Conference on Pattern Recognition, Hong-Kong, China, pages 573-576, 2006. 